Olivet Nazarene University Digital Commons@ Olivet

Faculty Scholarship - Mathematics

Mathematics

$3-1997$

\title{
Fibonacci Powers and a Fascinating Triangle
}

Dale K. Hathaway

Olivet Nazarene University, hathaway@olivet.edu

Stephen L. Brown

Olivet Nazarene University, sbrown@olivet.edu

Follow this and additional works at: https://digitalcommons.olivet.edu/math_facp

Part of the Numerical Analysis and Computation Commons

\section{Recommended Citation}

Hathaway, Dale K., and Stephen L. Brown. "Fibonacci Powers and a Fascinating Triangle." The College Mathematics Journal 28:2 (1997): 124-128.

This Article is brought to you for free and open access by the Mathematics at Digital Commons @ Olivet. It has been accepted for inclusion in Faculty Scholarship - Mathematics by an authorized administrator of Digital Commons @ Olivet. For more information, please contact digitalcommons@olivet.edu. 


\section{Fibonacci Powers and a Fascinating Triangle}

by Dale K. Hathaway and Stephen L. Brown

The Fibonacci sequence can be used as a starting point for an interesting project or research experience in a discrete mathematics class. The recurrence relation for the Fibonacci sequence is well known:

$$
F_{n}=F_{n-1}+F_{n-2},
$$

for $n>1$ with $F_{0}=1$ and $F_{1}=1$. Less well known is that there is a recurrence relation for $F_{n}^{2}$, which can be found in at least two different ways.

Our first method involves writing the Fibonacci sequence in terms of its explicit formula

$$
F_{n}=\frac{1}{\sqrt{5}} \alpha^{n+1}-\frac{1}{\sqrt{5}} \beta^{n+1}
$$

for $n \geq 0$, where $\alpha=(1+\sqrt{5}) / 2$ and $\beta=(1-\sqrt{5}) / 2$ [5], [6]. Squaring both sides results in an expression for $F_{n}^{2}$ :

$$
F_{n}^{2}=\frac{1}{5}\left[\left(\alpha^{2}\right)^{n+1}-2(\alpha \beta)^{n+1}+\left(\beta^{2}\right)^{n+1}\right]=\frac{1}{5}\left[\left(\alpha^{2}\right)^{n+1}-2(-1)^{n+1}+\left(\beta^{2}\right)^{n+1}\right] .
$$

Note that $F_{n}^{2}$ is a sum of three terms, whereas $F_{n}$ was a sum of two terms. That is, $F_{n}^{2}$ is a linear combination of the $(n+1)$ st power of $-1, \alpha^{2}$, and $\beta^{2}$, the roots of $(t+1)\left(t-\alpha^{2}\right)\left(t-\beta^{2}\right)=0$, or $t^{3}-2 t^{2}-2 t+1=0$. This yields the recurrence relation

$$
F_{n}^{2}=2 F_{n-1}^{2}+2 F_{n-2}^{2}-F_{n-3}^{2} .
$$

Another way to find this recurrence relation is by solving the system of equations

$$
\begin{aligned}
& 3^{2}=2^{2} a+1^{2} b+1^{2} c \\
& 5^{2}=3^{2} a+2^{2} b+1^{2} c \\
& 8^{2}=5^{2} a+3^{2} b+2^{2} c .
\end{aligned}
$$

A drawback of this second approach is that the student needs to know in advance that the recurrence relation involves the previous three terms, rather than just the previous two.

A modified Fibonomial triangle. Either approach can be extended to find recurrence relations for $F_{n}^{3}, F_{n}^{4}, \ldots$. To appreciate the resulting patterns, we have our students calculate all the recurrence relations through $F_{n}^{7}$. Using a computer algebra system (Matlab, Derive, Maple, etc.) or graphing calculator, they can zip through some messy computations and focus on the resulting patterns.

The student now has a sequence of equations, beginning with

$$
\begin{gathered}
F_{n}=F_{n-1}+F_{n-2} \\
F_{n}^{2}=2 F_{n-1}^{2}+2 F_{n-2}^{2}-F_{n-3}^{2} \\
F_{n}^{3}=3 F_{n-1}^{3}+6 F_{n-2}^{3}-3 F_{n-3}^{3}-F_{n-4}^{3} \\
F_{n}^{4}=5 F_{n-1}^{4}+15 F_{n-2}^{4}-15 F_{n-3}^{4}-5 F_{n-4}^{4}+F_{n-5}^{4}
\end{gathered}
$$


If we include the equation for power zero, $F_{n}^{0}=1$, at the top and move the $F_{n}^{m}$ terms to the right-hand side of each equation, we obtain the coefficients set out in Figure 1 in a triangular scheme similar to Pascal's triangle. This triangle (a variation of the Fibonomial triangle, which has no negatives [1], [2]) has a wealth of patterns for students to uncover:

- Diagonal 1 gives the Fibonacci sequence.

- There is an alternating pattern of $+/-$ signs by double diagonals.

- Ignoring signs, there is a symmetry about the vertical axis through the middle of the triangle.

The adventurous student will soon discover many less obvious patterns as well.

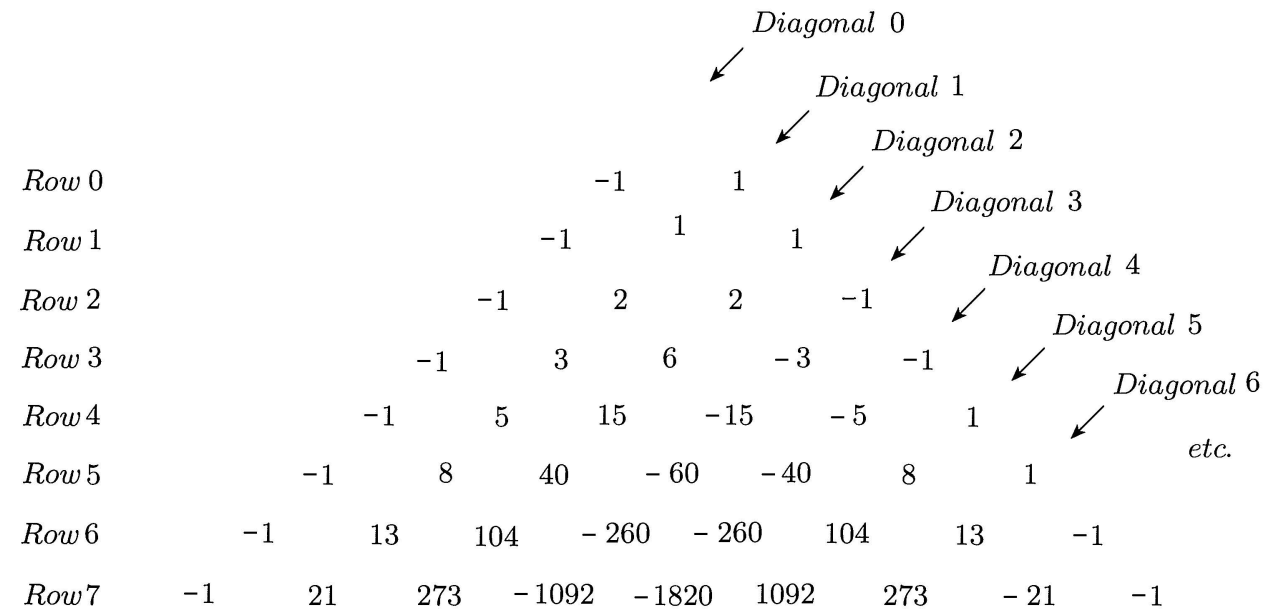

Figure 1. Modified Fibonomial triangle.

An important feature is uncovered if we examine how each entry is constructed from the previous entry in its diagonal by multiplying by a suitable fraction. Figure 2 shows the modified Fibonomial triangle with some multiplier values inserted. Do you see the pattern formed by the multipliers? The multipliers in diagonal $m$ are the ratios of Fibonacci numbers, $F_{n} / F_{n-m}$, where $n$ is the next row number!

Since each Fibonacci ratio is used as a multiplier to move down the diagonal one row at a time, these multipliers can be combined to yield an explicit formula for any entry by continually multiplying down a diagonal until the desired term is reached. For example, if we wish to find the value in the sixth row at the third diagonal, $F_{6,3}$, we multiply all the ratios on the third diagonal until we reach the sixth row:

$$
\begin{aligned}
F_{6,3} & =-1 \cdot \frac{F_{3}}{F_{0}} \cdot \frac{F_{4}}{F_{1}} \cdot \frac{F_{5}}{F_{2}} \cdot \frac{F_{6}}{F_{3}} \\
& =-1 \cdot \frac{3}{1} \cdot \frac{5}{1} \cdot \frac{8}{2} \cdot \frac{13}{3} \\
& =-260 .
\end{aligned}
$$


The general relationship is expressed by the formula

$$
F_{n, m}=(-1)^{\frac{m(m+1)-2}{2}} \cdot \prod_{k=1}^{n+1-m} \frac{F_{m+k-1}}{F_{k-1}} .
$$

Thus, each entry in the triangle can be found as a product of Fibonacci ratios.

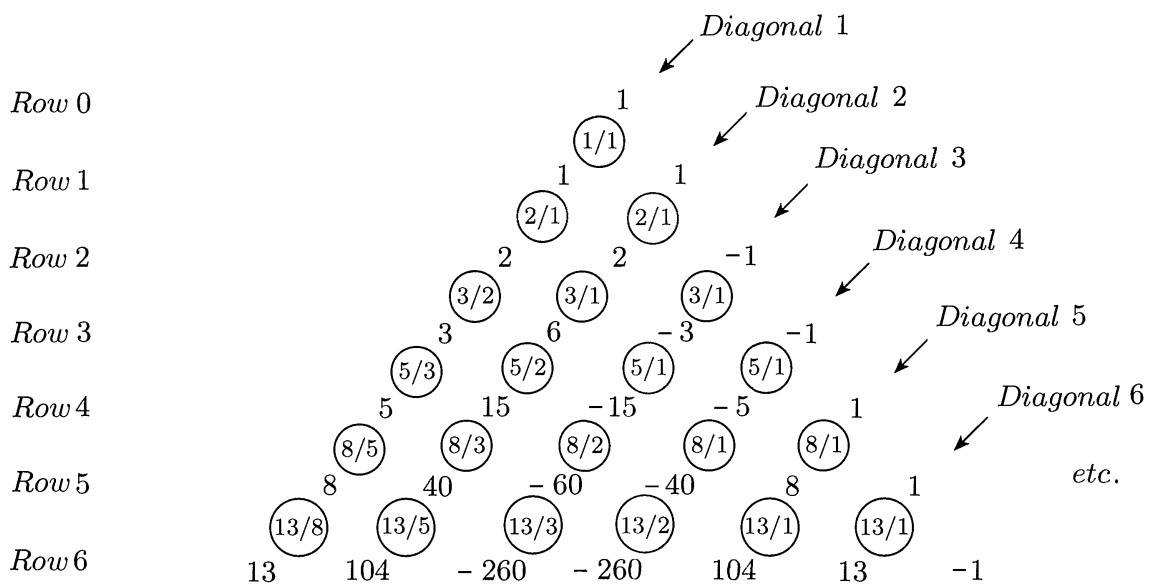

Figure 2. Modified Fibonomial triangle with multipliers circled (diagonal 0 is excluded).

It can be shown that the Lucas numbers also appear in the Fibonacci ratios $F_{n} / F_{n-m}$. The Lucas numbers, which follow the same recurrence formula as the Fibonacci numbers but with different initial conditions, are defined by

$$
L_{n}=L_{n-1}+L_{n-2}
$$

for $n>1$ with $L_{0}=1$ and $L_{1}=3$. The Fibonacci ratio used to obtain the middle entry of row $2 k+1$ is a Lucas number. In fact, since the middle entry of row $2 k+1$ is in diagonal $k+1$, the Fibonacci ratio used to obtain this entry is

$$
\begin{aligned}
\frac{F_{n}}{F_{n-m}} & =\frac{F_{2 k+1}}{F_{2 k+1-(k+1)}} \\
& =\frac{F_{2 k+1}}{F_{k}}
\end{aligned}
$$

which when reduced turns out to be $L_{k}$ [4].

Divisibility patterns. Pascal's triangle provides some interesting patterns of divisibility by various numbers [3], [7]. What is amazing is that the Fibonomial triangle also has some nearly identical patterns of divisibility by the same numbers! These patterns are especially noticeable for small prime number divisors, though some other divisors also show similarities. See Figure 3, where the evenly divisible entries are shaded. 

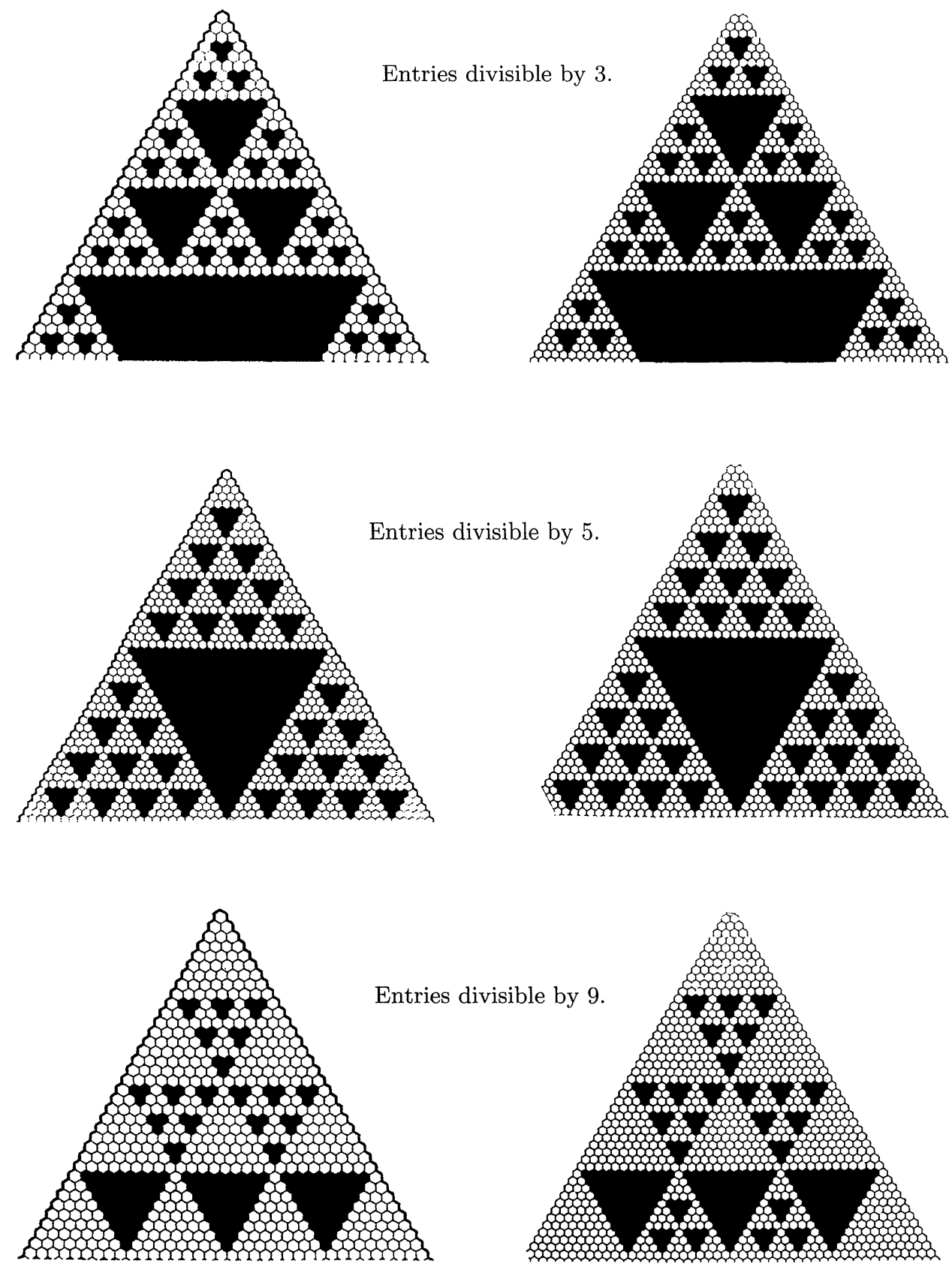

Figure 3. Divisibility of entries in Pascal's triangle (left) and the Fibonomial triangle (right).

The divisibility patterns are not always alike, however. Figure 4 shows the pattern of numbers divisible by 6 , where the disparity can easily be seen.

By building on some of these ideas, a student could develop a significant senior project investigating the powers of Fibonacci numbers. 

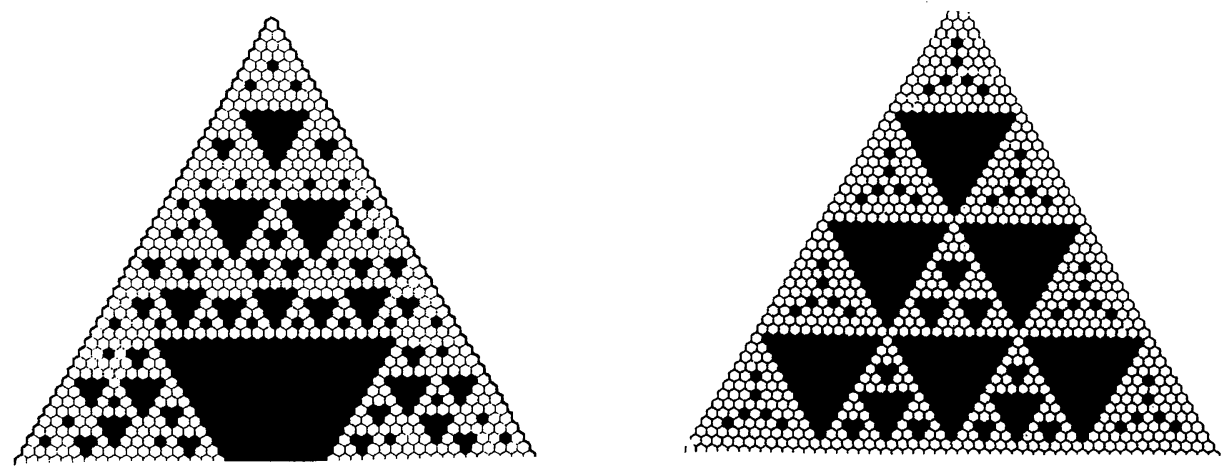

Figure 4. Entries divisible by 6 are shaded in Pascal's triangle (left) and the Fibonomial triangle (right).

Acknowledgments. The original catalyst for this project was a problem proposed by Stephen B. Maurer at a workshop organized by Marvin Brubaker on discrete mathematics at Messiah College in June 1992. We thank a referee for correcting a formula.

\section{References}

1. M. Bicknell and V. E. Hoggett, A Primer for the Fibonacci Numbers, Fibonacci Association, San Jose, CA, 1972.

2. T. A. Brennan, Fibonacci powers and Pascal's triangle in a Matrix, Part I, Fibonacci Quarterly 2 (1964) 93-103.

3. T. M. Green and C. L. Hamberg, Pascal's Triangle, Dale Seymour Publications, Palo Alto, CA, 1986.

4. V. E. Hoggett, Fibonacci and Lucas Numbers, Houghton Mifflin, Boston, MA, 1969.

5. R. Johnsonbaugh, Discrete Mathematics, Macmillan, New York, 1990.

6. S. B. Maurer and A. Ralston, Discrete Algorithmic Mathematics, Addison-Wesley, Reading, MA, 1991.

7. D. Seymour, Visual Patterns in Pascal's Triangle, Dale Seymour Publications, Palo Alto, CA, 1986. 